\title{
Erratum to: Risk factors for recurrence after transarterial chemoembolization for early-stage hepatocellular carcinoma
}

\author{
Hideaki Kinugasa $\cdot$ Kazuhiro Nouso $\cdot$ Yasuto Takeuchi $\cdot$ Tetsuya Yasunaka $\cdot$ Hideki Onishi \\ Shin-ichiro Nakamura $\cdot$ Hidenori Shiraha $\cdot$ Kenji Kuwaki $\cdot$ Hiroaki Hagihara • \\ Fusao Ikeda $\cdot$ Yasuhiro Miyake $\cdot$ Akinobu Takaki $\cdot$ Kazuhide Yamamoto
}

Published online: 15 December 2011

(C) Springer 2011

\section{Erratum to: J Gastroenterol \\ DOI 10.1007/s00535-011-0492-9}

Please note that errors appeared in the above-cited article.

In Abstract, in the "Results" section the first sentence should read: "The local recurrence rates and intrahepatic distant recurrence rates at 3 months, 6 months, and 1 year were 18.6, 33.4, and $61.8 \%$, and $2.8,2.8$, and $34.3 \%$, respectively."

In the main text, in the Results section, under the heading "Recurrence rate", in the second paragraph the second sentence should read: "The local recurrence rates and intrahepatic distant recurrence rates at 3 months, 6 months, and 1 year were 18.6, 33.4, and $61.8 \%$, and 2.8, 2.8 , and $34.3 \%$, respectively (Fig. 1)."

In the legend for Fig. 1, the second sentence should read: "The local recurrence rates (solid line) and intrahepatic distant recurrence rates (dotted line) at 3 months, 6 months, and 1 year were 18.6, 33.4, and $61.8 \%$, and 2.8, 2.8 , and $34.3 \%$, respectively."

The online version of the original article can be found under doi:10.1007/s00535-011-0492-9.

H. Kinugasa $(\varangle) \cdot$ K. Nouso · Y. Takeuchi · T. Yasunaka ·

H. Onishi - S. Nakamura $\cdot$ H. Shiraha $\cdot$ K. Kuwaki ·

H. Hagihara - F. Ikeda - Y. Miyake - A. Takaki - K. Yamamoto

Department of Gastroenterology and Hepatology,

Okayama University Graduate School of Medicine, Dentistry,

and Pharmaceutical Sciences, 2-5-1 Shikata-cho,

Kita-ku, Okayama 700-8558, Japan

e-mail: gyacy14@gmail.com 